\title{
ORAL GYMNASTICS- PERIODONTAL VANTAGE POINT
}

\author{
Dr Venkat Raghavan R, Dr. Anil Melath, Dr. Subair K, Dr. Arjun MR
}

Mahe Institute of Dental Sciences and Hospital, Chalakkara, Pallor, 673310, Mahe, India

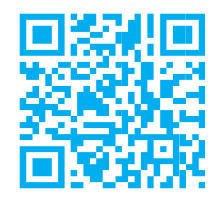

DOI: 10.37841/jidam_2021_V8_I3_05

Address for Correspondence

Dr Venkat Raghavan R,

Post-Graduate Student,

Mahe Institute of Dental Sciences and Hospital,

Chalakkara, Pallor , 673310, Mahe, India

E-Mail: dr.venkatraghavan94@gmail.com

Received: 26.08.2021

First Published: 11.09.2021

Accepted: 07.09.2021

Published: 27.9.2021

\section{ABSTRACT}

Facial musculature aids in orofacial functions and involves speech, mastication, deglutition. Orofacial myofunctional exercises are exercises which are associated with form and function of face and lip. This includes facial and tongue exercises such that it enhances proper tongue position, improved breathing, chewing and swallowing. Orofacial muscle exercises not only guide in management of deleterious oral habits but also improves the muscular tonicity and proper speech which leads to a congruous orofacial functioning. This article not only addresses oral cavity but also throws light over myofacial therapy that includes head and neck. It also facilitates proper nasal breathing, promotes post-operative healing by myofunctional exercise.

KEYWORDS: Periodontal Myotherapy, Oral gymnastics, Muscular exercise 


\section{INTRODUCTION:}

Oral cavity consists of teeth and periodontal structures which are consistently under the influence of surrounding musculature. This equilibrium mechanism which can control the teeth and supporting structures can be achieved through perioral muscles and tongue position. Any imbalance in the equilibrium alters the balance and leads to malocclusion. Various circumoral exercises help to intercept aberrant muscle activity and the resultant malocclusion $^{1}$.

Tongue is an influential organ which aids in articulation, deglutition, speech and various movements like protrusion and grooving. When properly positioned, the frenum enables the person to pronounce the alphabets properly and aids in phonetics. The position of tongue tip, not only leads to restriction of movements, also causes midline diastema between incisor and leads to gingival pull leading to recession of gingiva in the anterior and posterior regions ${ }^{2}$.

Ankyloglossia was foremost used in 1960s in the medical literature, when Wallace defined tongue-tie as "a condition in which the tip of the tongue cannot be protruded beyond the lower incisor teeth because of a short frenulum lingua, often containing scar tissue" ${ }^{2}$. The normal range of free tongue is greater than $16 \mathrm{~mm}^{3}$.

This review article throws light on gymnastic/myofunctional exercise in a specified way to achieve proper form, correcting dysfunctional patterns of habits, function, also aids in stability of surrounding hard/soft tissues.

\section{GOALS OF MUSCLE EXERCISES}

Major goal of this muscle exercise/gymnastic is to achieve lip seal, palatal tongue rest position, nasal breathing and an uninterrupted deglutition, also in dysfunctional habit corrections. Muscle exercise/gymnastic is a functional therapy in specific way in which muscles are trained to attain proper function, form along with the stability of surrounding hard and soft tissues. Muscle exercises are divided into four major types: lip exercises, tongue exercises, masticatory muscle exercises and breathing exercises.

\section{TONGUE EXERCISES}

'Tongue thrust' was considered to be a major causative factor of open bite and myotherapy was recommended to overcome this abnormal behaviour pattern ${ }^{4}$. Some of earlier studies investigate relationship between tongue and jaw movements. (Crompton et al., 1977; Oron and Crompton, 1985; Thexton and McGarrick, 1989; German and Franks, 1991). Anatomically genioglossus muscles is a fan shaped muscle which aids in relation with movements of jaw and lip activity during masticatory effects is still not understood ${ }^{5}$.

Abnormal tongue habit leads to malocclusion. Myofunctional therapy is indicated in severe situations as soon as the child is able to understand the problem and cooperate with the therapist. Intervening such habits by myotherapeutic tongue exercises and training in correct swallowing techniques as early as possible is one of the recommended methods for curbing the habit in most patients ${ }^{6}$.

One of the effective exercise to intercept tongue thrusting habit is $4 \mathrm{~s}$ exercise. This exercise identifies tongue spot, salivation, swallowing, and squeezing spot. The 4 steps are as follows:

1) Spotting exercise (1S) - Spot should be the rest position of the tongue.

2) Salivation exercise (2S) - The tongue is placed on the spot, which results in salivation.

3) Squeezing exercise (3S) - The tongue is squeezed vigorously with the teeth closed against the spot followed by relaxing.

4) Swallowing exercise (4S) - After squeezing, the next step is to swallow the spot. This new swallowing pattern should be practiced at least 40 times a day.

Reflex control is uptake at subconscious level in this mode of exercise, to reinforce new swallowing pattern. Patient is instructed to concentrate on handheld clock and note down the duration of holding the table in accurate position. This is to mainly divert the concentration. Now ask the patient to hold the citric acid tablet with biconcave surface using the tongue tip against the hard and soft palate with gradual increase in duration.

Another method commonly known as ELASTIC EXERCISE, it may be one or two elastic swallows. By this way patient can be guided about the proper tongue posture during swallowing ${ }^{6}$ (Fig 1).

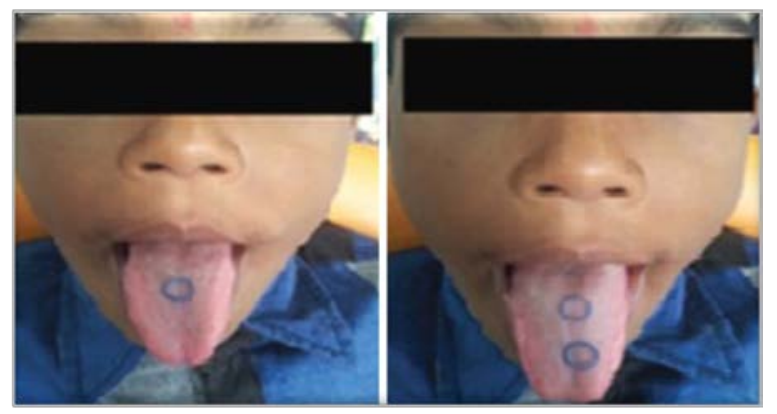

Fig 1: ELASTIC EXERCISE-with one or two elastics swallow 
"Hold pull exercise" is another type in which, tip of the tongue contacts papilla at the crest of hard palate. Then the patient is asked to gradually open the mouth without the tongue losing the contact with palate. This exercise helps in stretching the lingual frenum ${ }^{7}$ (Fig 2).
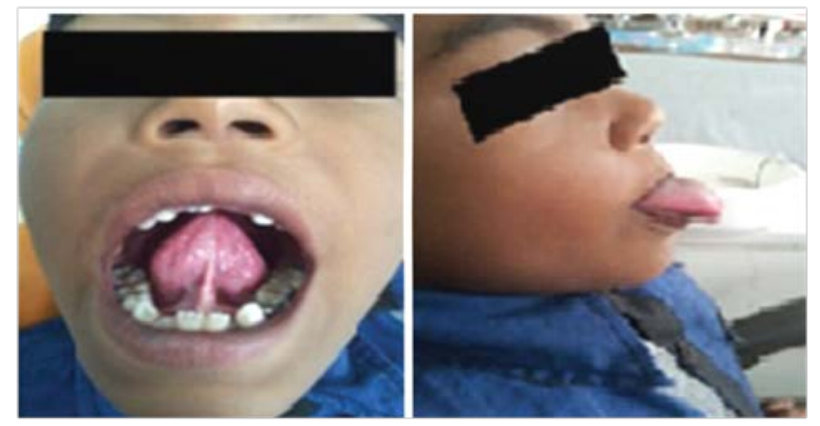

Fig 2: Hold pull Exercise-Tip of the tongue contact with the crest of hard palate.

"Tongue retraction exercise" which is performed by palate of the mouth touched by back of tongue in position for upto 3 seconds. This exercise is repeated for about 5 times. Tongue extension exercise is performed by protruding the tongue between lips and holding it steady and straight for 3-5 s. It is repeated for about 5 times.

\section{HABIT BREAKING EXERCISES}

Various persistent deleterious habits lead to various etiological problems starting from sinus problems to sleep apnea, adenoids or even enlarged tonsils. These etiological factors are by deleterious habits such as mouth breathing. This type of breathing is dangerous, increasing the risk of coronary disease, anxiety and dental problems in children. To get rid of mouth breathing, one can take steps at home that will help in retraining oneself to breathe through the nose instead of the mouth. There are 3 main steps to be performed.

i. In the first step, allergy and cold should be treated as a primary concern. Blockage in the nasal passage worsens mouth breathing, especially during sleeping hours.

ii. In the second step, nose breathing, that is physiological breathing should be practiced from childhood. This can be achieved by positioning the mouth in a relaxed closing way and rapid inhalation and exhalation is performed through the nose. This is tried for three in-and-out breath cycles per second while it promotes a proper breathing technique.

iii. Final step is to sleep on the back. This opens the airways and takes pressure off the sinus cavities, stimulating air exchange through the nose instead of the mouth.

\section{BREATHING EXERCISES}

During growth spurt period, facial appearance and morphology have huge impact by deleterious habits like mouth breathing. Six muscle groups (i.e., bilateral masseter, temporalis, and orbicularis oris) were monitored to allow a better examination of absolute and relative muscle activity. Two groups of masticatory muscles (i.e., lateral and medial pterygoids) were not included due to the need for intrusive procedures (i.e., needle electrodes) to record activity from these sites ${ }^{8}$. Nasal breathing apart from enabling an adequate growth and development of the craniofacial morphology also interacts with other physiological functions such as chewing and swallowing. Major three breathing exercises are pranayama, blowing balloon and holding water in the mouth and breathing through the nose.

In pranayama exercise, breathing is performed through nose by rapid inhalation and exhalation. During this exercise mouth should be closed in a relaxing position. Three in-and-out breath cycles per second should be tried and done(Fig 3).

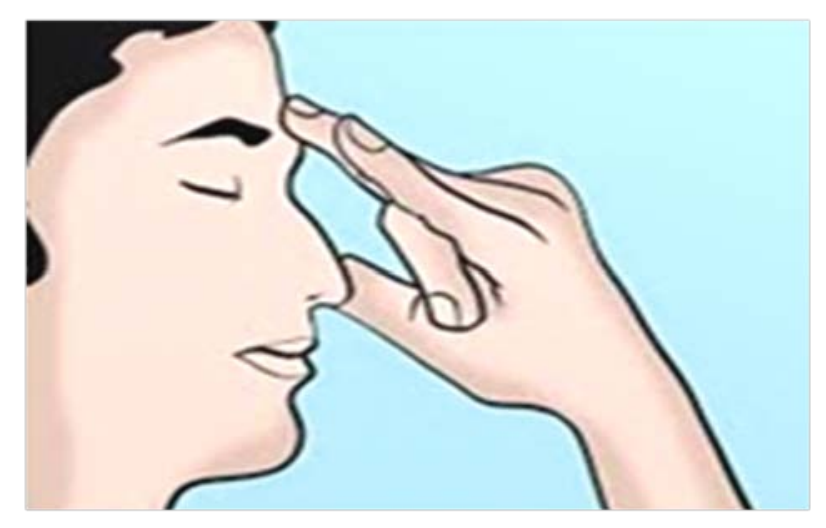

Fig 3: Pranayama Exercise.

Another way of exercise is Blowing of balloon. This performed by deep inhalation by nose followed by an exhalation through mouth. Balloon in the mouth and repeat the breathing exercise until 5 breaths of exhalation into the balloon ${ }^{9}$.

\section{CHEEK EXERCISES}

In this cheek exercise, water is held inside mouth and side to side movements started. It is not only done to improve the tonicity of perioral muscles, but also improves lateralization and coordination of tongue movements. Furthermore, the tongue is rolled from rightleft/left-right cheek(Fig 4). 


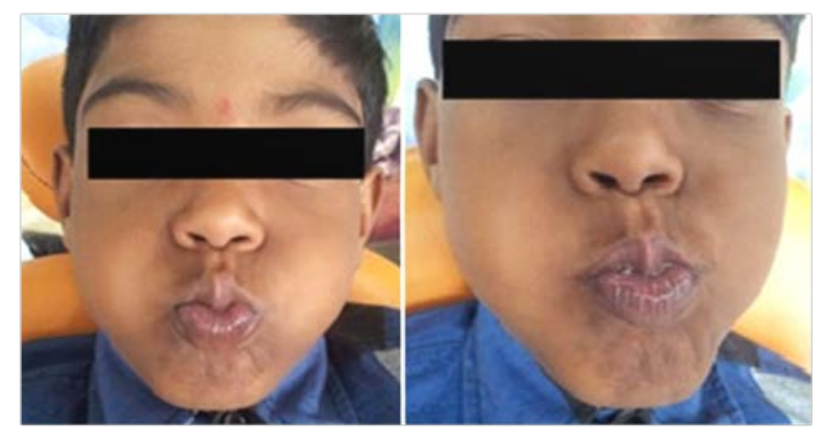

Fig 4: Cheek Exercise- Improves tonicity of peri-oral muscles/tongue movements co-ordination.

Another added exercise is puffing of cheeks in which the cheeks are filled with air, and the air is moved from one cheek to the other, duration of about 5-10 times. During this exercise no amount of air should be let free from round lips or nose. It is repeated for about 5 times.

\section{EXERCISES BY WIND INSTRUMENTS}

Music as a therapy is way back 4000 years ago where Egyptian priest doctors used an incantation which was intended to have a positive influence over pregnant women. Hippocrates in 400 B.C. took his mental patients to the temple to listen to music ${ }^{10}$ Playing wind instruments crucially depends on the ability to control the orofacial muscles and actually may be an interceptive orthodontic procedure. Both vocal and wind instrument sound production entail the use of involved facial muscles, for example, in the movement patterns of lips, jaw, and tongue during the articulation. Therefore, motor control of these structures is a key component of sound production for both vocal and wind instrument performance $^{11}$.

Use of trumpet will help in strengthening the lips and cause the tongue to confine its action within a definite area. The area of lips, which come in contact with a mouthpiece will feel a stimulating effect and fuller flow of blood to the musculature. By continued use of this instrument hypotoned tissue will develop a normal tonicity and short flabby lips will lengthen. During the use of this instrument, the tongue is raised from the floor of the mouth and stimulates the tissue of lower lip and reduces tension in the upper lip.

For playing Flute, the lower lip rolls over the side of the head of the instrument and upper lip are stretched downward, and the air is directed into the instrument is useful in those cases where patient is having short upper lip; strong muscle activity and protruding lower lip.

\section{CONCLUSION}

Oral musculature exercise is a sparse topic in field of Periodontics. A change in muscular activity can initiate not only morphological variations but also aids in form, functions, aesthetics of teeth and supporting bone. Due to its compensatory or adaptive myogenic activity and functions it also aids in healing after periodontal procedures like lingual frenectomy. Orofacial muscle exercise through these simple and effective methods helps in favorable lip seal along with other muscular changes, thereby assisting in eliminating most deleterious habits, and reducing complex surgical procedures.

\section{FINANCIAL SUPPORT AND SPONSORSHIP}

Nil

\section{CONFLICTS OF INTEREST}

There are no conflicts of interest.

\section{REFERENCES}

1. Sakshi Khemka, Nilima Thosar, Sudhindra Baliga; Oral gymnastics - Way to a harmonious dentition; International Journal of Contemporary Dental and Medical Reviews (2015)

2. Abhishek Singh, Mala Dixit Baburaj and Sandeep Pimpale; Management of Ankyloglossia by Frenectomy; ACTA SCIENTIFIC DENTAL SCIENCES; 2019 January; Volume 3 Issue 1

3. Kotlow LA; “Ankyloglossia (tongue-tie): A diagnostic and treatment quandary”. Quintessence International 30 (1999): 259-262.

4. Rolf Frankel; Lip seal training in the treatment of skeletal open bite; European Journal of Orthodontics 2 (1980) 219-228

5. K. Takada, K. Yashiro, Y. Sorihashi, T. Morimotol, and M. Sakuda; Tongue, Jaw, and Lip Muscle Activity and Jaw Movement during Experimental Chewing Efforts in Man; J Dent Res 1996; August;75(8): 1598-1606,

6. Chawla Hs., Suri S, Utreja A; Is tongue thrust that develops during orthodontic treatment an unrecognized potential road block? J Indian Soc Pedod Prev Dent - 2006; June ;80-83.

7. Phulari BS, Bhagwandass AR. Interceptive orthodontics. In: Phulari BS, editor. Orthodontics: Principles and Practice. 1st ed. New Delhi: Jaypee Brothers; 2011. p. 261.

8. Moss Ra, Villarosa Ga, Cooley, Lombardo Tw; Masticatory Muscle Activity as A Function of Parafunctional, Active and Passive Oral Behavioural Patterns; Journal of Oral Rehabilitation; 1981; Volume 14, Pages 361-370

9. KyoChul Seo,;MiSuk Cho; The effects of a balloonblowing exercise in a 90/90 bridge position using a ball on the pulmonary function of females in their twenties; J Phys Ther Sci. 2018 Oct; 30(10): 12671270. 
10. George E. Dillinger; Music and Music Therapy as a Patient Experiences Them; Music Therapy 1958 Eighth Book of Proceedings of The National Association for Music Therapy, Inc.Volume Viii; P188-194.

11. Strayer ER. Muscle instruments as an aid in the treatment of muscle defects and perversions. Angle Orthod 1938; 9:18-27. 